\title{
Effect of nitrate or sulfate on flocculation properties of cationic polymer flocculants
}

\author{
Binghui Tian*, Xiaopeng Ge, Gang Pan, Zhaokun Luan \\ State Key Laboratory of Environmental Aquatic Chemistry, Research Center for Eco-Environmental Sciences, \\ Chinese Academy of Sciences, Beijing 100085 \\ Tel./Fax:+86(10)62943436; email: tbh_8@163.com
}

Received 10 November 2005; Accepted 3 April 2006

\begin{abstract}
The objective of this study was to investigate the effect of different counterions on the flocculation of cationic polymer flocculants and to obtain new high efficient flocculants by modifying polydiallyldimethylammonium chloride (PDADMAC) with different counterions. The effect of nitrate or sulfate as counterions on flocculation properties of polydiallyldimethylammonium salts (PDADMAX) was investigated in the conventional jar test procedure by using optical monitoring technique and zeta potential measurement. Moreover, in order to indicate the flocculation mechanism of PDADMAX having different counterions, the effect of counterions on the solution property and adsorption conformation of PDADMAX was studied by reduced viscosity and atomic force microscopy (AFM). The experiment results show that counterions have a significantly different effect on PDADMAX flocculation where "adsorption charge neutralization action" and "adsorption bridging action" play different parts. PDADMAX having $\mathrm{NO}_{3}^{-}$has stronger "adsorption charge neutralization action" and its turbidity removal is $98 \%$, whereas PDADMAX having $\mathrm{SO}_{4}^{2-}$ has stronger "adsorption bridging action", as well as the wider optimum dosage and larger floc size. In particular, the flocculation efficiency of PDADMAX having different contents of $\mathrm{SO}_{4}^{2-}$ or $\mathrm{NO}_{3}^{-}$ is different. The optimum content of $\mathrm{SO}_{4}^{2-}$ or $\mathrm{NO}_{3}^{-}$is $20 \%$.
\end{abstract}

Keywords: Polydiallyldimethylammonium salts; Counterion content; Nitrate; Sulfate; Flocculation; Atomic force microscope

\section{Introduction}

Flocculation is one of the most important decontamination processes in drinking and waste-

*Corresponding author. water treatment. Flocculant is undoubtedly the most important factor in this process, and high performance flocculants are constantly sought. In recent years, cationic, quaternary ammonium polyelectrolytes have the subject of increased

0011-9164/07/\$- See front matter (C 2007 Published by Elsevier B.V.

doi:10.1016/j.desal.2006.04.077 


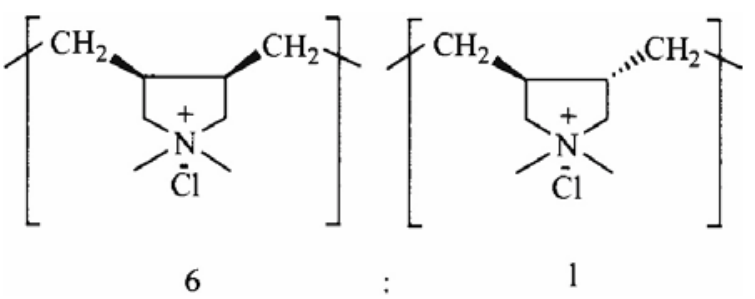

Fig. 1. Chemical structure of PDADMAC.

research efforts because of their diverse commercial applications [1,2].

Polymers of diallyldimethyl ammonium chloride (PDADMAC) (the chemical structure of PDADMAC can be seen in Fig. 1) are the most prominent water-soluble cationic ammonium polymers. They have been widely used as primary flocculants or flocculant aids in destabilization of colloidal suspensions, clarification of industrial wastewaters, separation of solid-liquid systems, treatment of sewage, and so forth $[3,4]$. However, comparing with other polymer flocculants, PDADMAC is difficult to have high molecular weight, which will make against "adsorption bridging action" of PDADMAC when it is used as a flocculant in water treatment $[5,6]$. To improve "adsorption bridging action" or reduce the running costs of the PDADMAC flocculants, some modification techniques and preparation methods such as copolymerization with hydrophobic and hydrophilic monomers [7,8], polyelectrolyte complexes (PECs) formed between oppositely charged macromolecules [3,9] and cationic particles prepared by polymer being adsorbed or grafted on clay [10,11] have been reported in recent years. But the above methods cannot resolve the key problem of PDADMAC flocculants as cationic polymer flocculants, which are hoped to have both high molecular weight and high cationic density, namely, have both stronger "adsorption charge neutralization action" and stronger "adsorption bridging action".

For cationic polymer flocculants, besides molecular weight and cationic density, some other factors such as $\mathrm{pH}$, ionic strength and counterions have a great effect on their solution and flocculation behavior. In the previous literature $[5,6]$, it was found that when there is sulfate ion in the polymer solution, at low ionic strengths, electrophoretic mobility and dilute solution viscosity of PDADMAC are higher at low $\mathrm{pH}$ than those at high $\mathrm{pH}$ [12-14]. The $\mathrm{pH}$ dependent conformational changes are then attributable to the sulfate ion. The reasons of the above problem are that the physicochemical properties of polyelectrolyte solutions depend crucially on the strength of coulombic interactions between the counter-ions and the charged groups of the functional polymer. The anion binding of PDADMAC appears to be intramolecular at low sulfate concentrations and intermolecular at high sulfate concentrations [14,15]. On the other hand, the previous works still indicated that besides the characters of cationic polymer flocculant, the particle property, especially electrostatic interactions on solid-liquid interfaces and macromolecular dynamics in the solution also play a significant role on the flocculation mechanism of cationic polymer flocculants. The causes are that the flocculation mechanism of cationic polymer flocculation does not only depend on the adsorption quantity of polyelectrolyte chains, but also depends on the adsorption structure and conformation of cationic polymer [5].

In the flocculation application, cationic polymer flocculants are known to bind to the particle surface in different conformations - "loops", "trains", and "tails". Many factors can affect the adsorption conformation of cationic polymer flocculants at a particle surface. For example, the cationic polymer with high charge densities adsorbed flat and flocculated via the charge neutralization or patch mechanism, while polyelectrolytes with low charge densities formed "loops and tails" and flocculated by a bridging 
mechanism [18]. Therefore, for cationic polymer flocculants, studies on their adsorption conformation are of great scientific and practical interest.

A range of experimental techniques, including optical reflection and scattering technique, electrochemical and quartz crystal microbalance (QCM), neutron reflectivity, X-ray reflectivity, hydrodynamic techniques, and electron microscopy, etc. [16-21] have been used to characterize the adsorption conformation and adsorption mechanism of polyelectrolytes (e.g., PDADMAX salts, etc.). However, in low ionic strength solutions, polyelectrolytes adsorbed at an oppositely charged surface forms thin layers. Due to the limited screening of the electrostatic charges on the polymer, long-range lateral repulsion can cause a heterogeneous surface coverage [22]. So the above methods cannot provide a direct proof for the conformation of polymer absorbed at a surface. To obtain detailed information on effects of counterions on absorption and flocculation properties of PDADMAX, such as adsorbed layer structure or the lateral distribution of cationic polymer flocculants, one may preferentially use the atomic force microscopy (AFM) force volume technique [23].

In the previous works, many studies were mainly interested in the investigation of the influence of the counterions on mobility and solution properties of polyelectrolytes. It has been found that the polycation mobility is dependent on the counterion nature, which may in turn affect the adsorption and flocculation behaviors of the polyelectrolyte. However, few paper studies the effect of the counterions on the flocculation behaviors of cationic polymer flocculants [23,24]. In this paper, polydiallyldimethylammonium nitrate (PDADMANO $\left.{ }_{3}\right)$ and sulfate $\left(\mathrm{PDADMASO}_{4}\right)$, with a high molecular weight and different contents of counterions, were prepared from PDADMAC. The solution properties of PDADMA salts (PDADMAX) having different counterions, as well as their flocculation behaviors for kaolin suspension, were investigated by viscometry, residual turbidity, distributions of zeta potential and flocculation index of kaolin suspension. AFM imaging was further performed to characterize their adsorption conformation on the surface of oppositely charged mica.

\section{Experimental}

\subsection{Reagents}

A 40 wt.\% of PDADMAC aqueous solution (Florage, SNF, France) was purified using a Pellicon $^{\mathrm{TM}}$ ultrafiltration system equipped a Millipore $^{\mathrm{TM}}$ tangential flow filter membrane with a molecular weight cut-off (MWCO) of $100 \mathrm{KD}$, and freeze dried. Its molecular weight was determined to be $0.48 \times 10^{4}$ using the viscometrical method $\left(1.0 \mathrm{M} \mathrm{NaCl}, 25,[\eta]\left(\mathrm{ml} \times \mathrm{g}^{-1}\right)=1.12 \times\right.$ $10^{-4} \mathrm{M}^{0.82}$ according to the Mark-HouwinkSacurada equation [25].

Silver salts of nitrate and sulfate were of analytical reagent grade (Beijing Chemical Reagent Agent, China). Double distilled water was used for the test.

\subsection{Preparation of $\mathrm{PDADMANO}_{3}$ and $\mathrm{PDADMASO}_{4}$}

Ten ml PDADMAC aqueous solution ( $0.3 \mathrm{~mol} / \mathrm{L}$, expressed in terms of monometric units) was mixed with the stoichiometric amounts of silver salt of nitrate or sulfate under stirring. After $0.5 \mathrm{~h}$ of reaction, the suspension solution was centrifuged and the supernatant was freezedried. The contents of $\mathrm{NO}_{3}^{-}$and $\mathrm{SO}_{4}^{2-}$ and the residual $\mathrm{Cl}^{-}$as well in the prepared polydiallyldimethylammonium salts were measured by Ion Chromatography 4500i (Dionex CO., USA), and the counterions contents were found for $\mathrm{NO}_{3}^{-}$ 19.5\% 39.1\% 59.6\% 79.3\% 99.5; $\mathrm{SO}_{4}^{2-} 19.1 \%$ $39.6 \% 59.4 \% 79.7 \% 99.7 \%$. 
2.3. Characterization of the modified polymer flocculants of $\mathrm{PDADMANO} \mathrm{O}_{3}$ and $\mathrm{PDADMASO} \mathrm{H}_{4}$

\subsubsection{Reduced viscosity $\left(\eta_{s p} / c\right)$ measurement}

Viscometric measurements were carried out by an Ubbelohde viscometer $(4 \mathrm{ml}, 0.6 \mathrm{~mm})$ at $25 \pm 0.1$. All polymer solutions were made up of double-distilled water. The solutions were filtered by sand filter and kept overnight. Prior to the measurements the solutions were kept about $15 \mathrm{~min}$ at ambient for temperature equilibrium. The concentration $\left(C_{P}\right)$ of PDADMAX ranged from $2.5 \times 10^{-3}$ to $5.0 \times 10^{-2} \mathrm{~mol} / \mathrm{L}$. In this concentration region, the kinetic energy and shear rate were regarded as negligible, and the density of solution was considered to be approximately the same as that of pure water. According to Nishida et al. [26], the reduced viscosity $\left(\eta_{s p} / c\right)$ can be calculated in the following equation: $\eta_{\mathrm{SP}} / C=$ $\eta_{\text {intra }} / C+\eta_{\text {inter }} / C$ where $\eta_{\text {intra }} / C$ is the intramolecular hydrodynamic contribution, and $\eta_{\text {inter }} / C$ represents the intermolecular electrostatic contribution.

2.3.2. Observation of cationic polymer absorbed structures by atomic force microscopy

Atomic force microscopy (AFM) imaging was performed to characterize molecular conformations and adsorbed layer structures of the three PDADMA-X salts. The AFM used was a NanoScope Шa Multimode Scanning Probe Microscopy Instrument (Digital Instruments, Santa Barbara, CA) equipped with an E-scanner operating in air at room temperature with $50-60 \%$ humidity. A tapping mode was employed, and images were taken with a $256 \times 256$ data points (pixel resolution), acquired by using the retrace signal. The silicon cantilevers with $125 \mu \mathrm{m}$ in length and $300 \mathrm{KHz}$ in frequency (Nanoprobe, Model RTESP14) were employed. The scanning speed and the loop gain factors were varied during the imaging process. Line scan rates were typically set at $1.0 \mathrm{~Hz}$ for tapping mode with other scan parameters such as scan size, set point, integral gain, and proportional gain varied and optimized. In order to avoid tip-related artifact, imaging was performed with a minimal force, and image feature were reproduced before being accepted as representative. The drop deposition method was adopted for sample preparation, and a $0.02 \mathrm{ml}$ of polymer solution was deposited onto the freshly cleaved mica surface and dried $15 \mathrm{~min}$ at $20^{\circ} \mathrm{C}$ in a thermostated container. For each experimental condition, two mica sheets were prepared and at least three images were obtained for each mica sheet sample. Images were taken with online filtering and subsequently by flattening to remove the background slope. Using NanoScope Image analysis software, section analysis of images was conducted to measure the size of particles and their aggregates.

\subsection{Flocculation experiments}

A stock suspension of purified kaolin (Beijing Chemical Reagent Agent, China; composition of kaolin is $\mathrm{SiO}_{2} 50.1 \%, \mathrm{Fe}_{2} \mathrm{O}_{3} 0.28 \%, \mathrm{Al}_{2} \mathrm{O}_{3} 35.8 \%$, $\mathrm{K}_{2} \mathrm{O} \quad 0.05 \%, \mathrm{Na}_{2} \mathrm{O} 0.06 \%, \mathrm{CaO} 0.07 \%, \mathrm{MgO}$ $0.06 \%, \mathrm{TiO}_{2} 0.15 \%$ ) was prepared to a concentration of $100 \mathrm{~g} / \mathrm{L}$. Size distribution of the kaolin suspension was determined on a Laser Particle Analyser (Mastersizer 2000, Malvern CO., UK). $\mathrm{D}(0.9)$ of the particles was $2 \mu \mathrm{m}$, with a mean size of about $0.92 \mu \mathrm{m}$. Working suspensions were prepared by diluting the stock suspension in a test solution containing $5 \times 10^{-4} \mathrm{M}$ of both $\mathrm{NaNO}_{3}$ and $\mathrm{NaHCO}_{3}$ to provide fixed concentrations of electrolyte and alkalinity.

For flocculation experiments, a clay concentration with $1000 \mathrm{mg} / \mathrm{L}$ was used. The flocculation experiments were carried out in a $1 \mathrm{~L}$ cylindrical vessel, stirred by the flat-blade paddle. The flocculation experiments were conducted by $1 \mathrm{~min}$ of rapid mixing at $300 \mathrm{rpm}\left(G=143.5 \mathrm{~s}^{-1}\right)$ followed by $10 \mathrm{~min}$ of slow stirring at $40 \mathrm{rpm}$ $\left(G=9.5 \mathrm{~s}^{-1}\right)$. Flocs were allowed to settle in 
30 min. Residual turbidity (RT) and the zeta potential of fine particles remaining in the supernatant were measured on a HACH $2100 \mathrm{~N}$ Turbidimeter (Hach, Loveland, Co.) and a Zetasizer 2000 (Malvern CO., UK), respectively. Throughout the mixing and coagulation periods, the suspension was continuously sampled by peristaltic pump and monitored by a Photometric Dispersion Analyzer (PDA 2000; Rank Brothers) to obtain a flocculation index (FI). The FI has been clearly defined in the literature [27].

\section{Results and discussion}

\subsection{Observation of cationic polymer absorbed layer structures by AFM}

As a powerful tool for imaging surface structure at nanometer scale, AFM has been widely used in characterization of adsorbed layer structures of polymers [26]. In order to gain more detailed information on the structure and conformation of the polymer flocculants with different counterions $\left(\mathrm{Cl}^{-}, \mathrm{NO}_{3}^{-}\right.$and $\left.\mathrm{SO}_{4}^{2-}\right)$, molecular conformation and adsorbed layer structures of the three PDADMAX salts on mica were investigated by AFM at two different concentration levels, $5 \mathrm{mmol} / \mathrm{l}$ (Fig. 2a, c and e, left) and $0.05 \mathrm{mmol} / \mathrm{l}$ (Fig. 2b, d and f), respectively. As shown in Fig. 2, sponge-like membrane structures with a height of 2 to $4.5 \mathrm{~nm}$ were observed when $C_{p}=5 \mathrm{mmol} / \mathrm{l}$, showing little difference in absorbed layer morphology for all the three polymers (Fig. 2a, c and e). When $C_{p}=0.05 \mathrm{mmol} / \mathrm{L}$, however, the adsorption structures of PDADMAX are completely different. While a uniform hemispherical structure with $8-10 \mathrm{~nm}$ in height and $40-55 \mathrm{~nm}$ in width was found for $\mathrm{PDADMASO}_{4}$ (Fig. 2f), a pearl necklace-like aggregation structure of uniform hemispheroids with the height of 15 to $20 \mathrm{~nm}$ and width of 25 to $45 \mathrm{~nm}$ was observed for PDADMAC (Fig. 2b), and a sponge-like layer structure with the height of 1 to $5 \mathrm{~nm}$ was given for $\mathrm{PDADMANO}_{3}$ under the same experimental conditions (Fig. 2d). The polymer sizes of PDADMAX given by the AFM are very consistent with the data obtained by light scattering or by molecular dynamics calculation in literatures [29,2]; furthermore, the obtained structural features are of high uniformity. Such results infer that AFM is a valuable tool for studying on polymer adsorption at single molecular level.

The differences in adsorption conformation of the three polymers in dilute solution $\left(C_{p}=\right.$ $0.05 \mathrm{mmol} / \mathrm{L}$ ) can be attributable to the effect of the different counterions. It has been known that the physicochemical properties of polyelectrolyte solutions depend crucially on the strength of coulombic interactions between the counter-ions and the charged groups of the polyion. Polyelectrolytes usually develop a more extended structure in dilute solution at low ionic strengths, due to the mutual repulsions among the charged segments of the polyelectrolyte chain, and adsorbs at particle surfaces in a way mainly determined by the electrostatic interactions. As shown in Fig. 3, the electrostatic combination of the chloride counterions onto the polycation chain of PDADMAX lessens the charge density of the polycation chain and leads to a lower intramolecular repulsive interaction so that PDADMAC coils up and adsorbs itself on the oppositely charged mica surface with loops and tails. Moreover, the intermolecular interaction is not strong enough to completely inhibit the congregation among polymer chains of PDADMAX; therefore, a pearl necklace-like aggregation structure with a heterogeneous surface coverage was obtained for the PDADMAC (Fig. 3a). For the $\mathrm{PDADMASO}_{4}$, however, the bivalent sulfate counterions have a very strong electrostatic interaction on both the intramolecular attraction and the intermolecular repulsive forces, so that the extended polymer chains coil up into small compact spheres and 

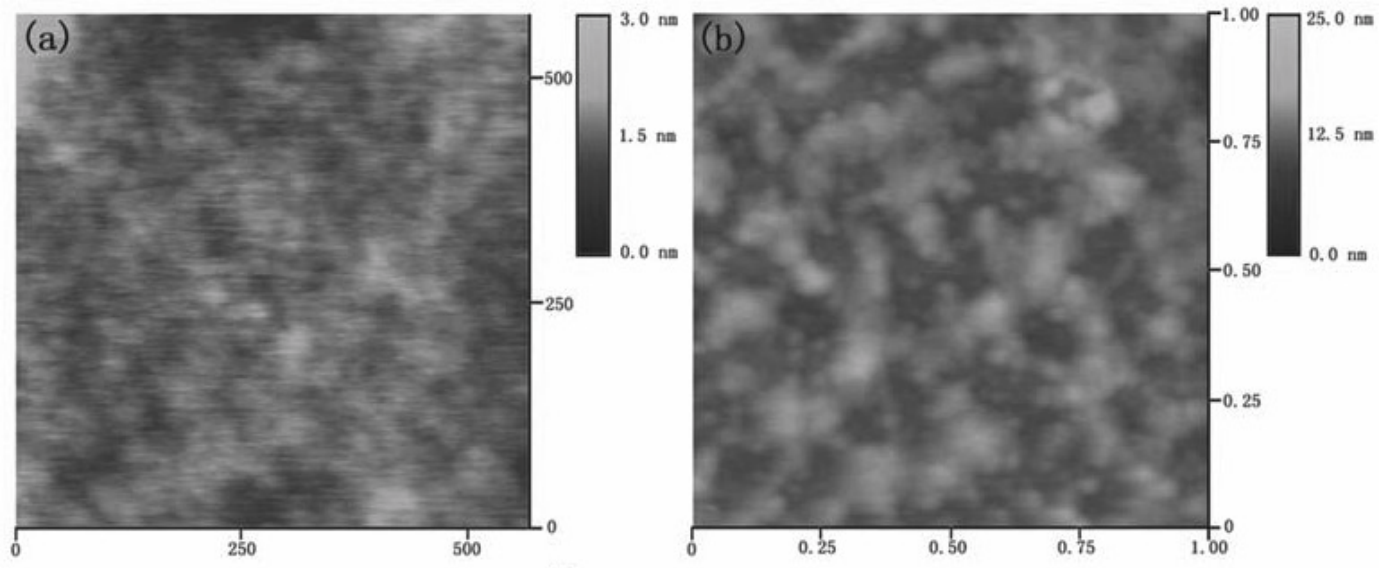

(a) $5 \mathrm{mmol} / \mathrm{L}$ PD ADMAC

$\mathrm{nm}$

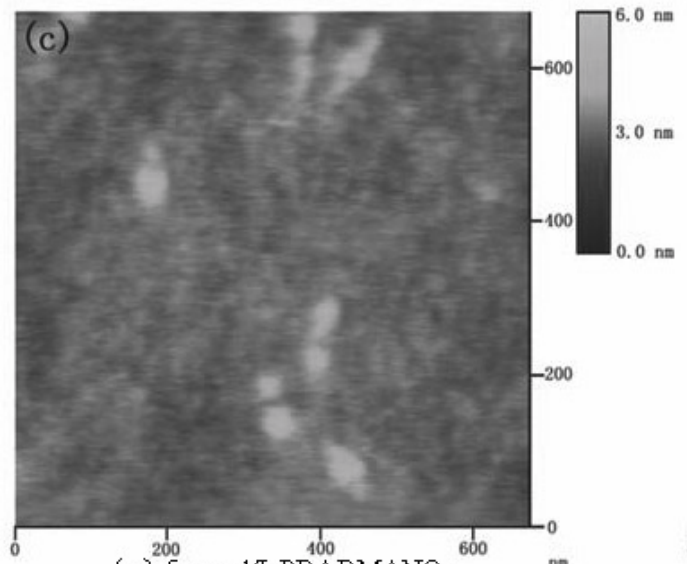

(b) $0.05 \mathrm{mmol} / \mathrm{L}$ PD ADMAC

$\mu \mathrm{m}$

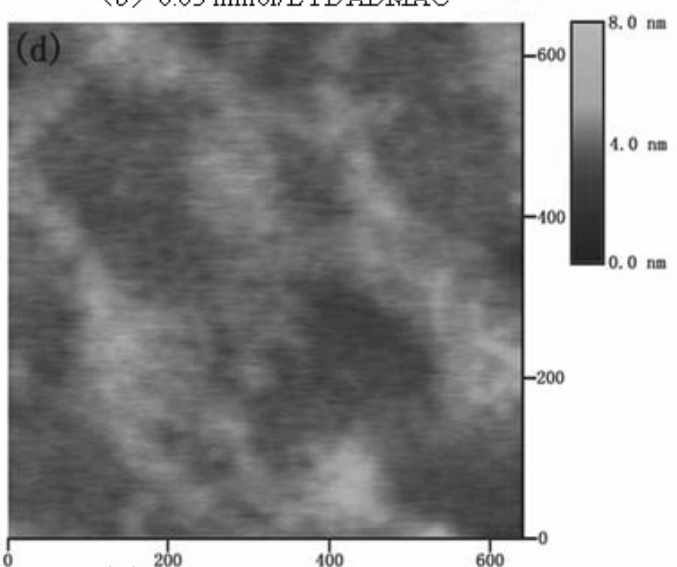

(d) $2005 \mathrm{mmol} / \mathrm{L}$ PD $400 \mathrm{AMANO}_{3}^{60}$

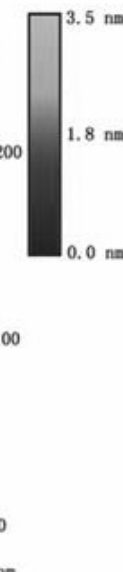

ne

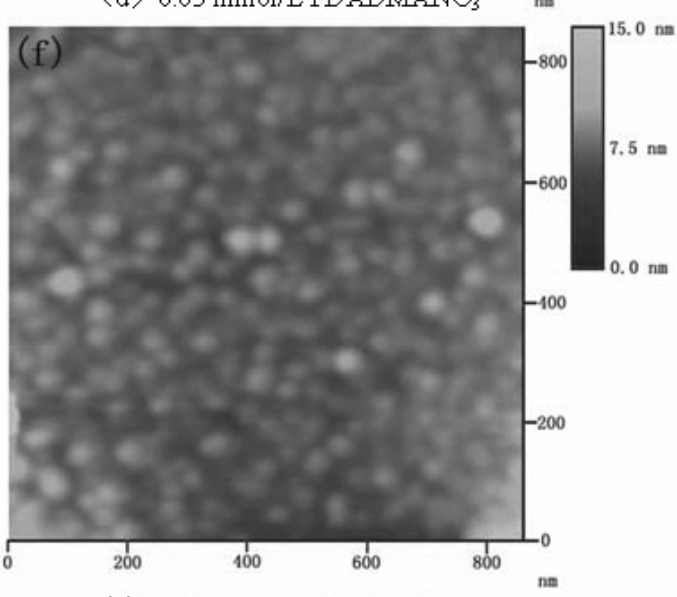

(f) $0.05 \mathrm{mmol} / \mathrm{L}$ PDADMASO

Fig. 1. AFM images of PDADMAC, $\mathrm{PDADMANO}_{3}$ and $\mathrm{PDADMASO}_{4}$ absorbed on the surfaces of oppositely charged mica from aqueous solution $\left(C_{\mathrm{s}}=1.0 \times 10^{-3} \mathrm{~mol} / \mathrm{l}, \mathrm{NaCl}\right)$ at two different concentration levels $\left(C_{p}\right)$ of $5 \mathrm{mmol} / 1$ (left: a, $\mathrm{c}$ and e) and $0.05 \mathrm{mmol} / \mathrm{l}$ (right: b, d and f), respectively. (a) and (b), PDADMAC; (c) and (d), PDADMANO ${ }_{3}$; (e) and (f), $\mathrm{PDADMASO}_{4}$. 
(a)

(b)

(c)

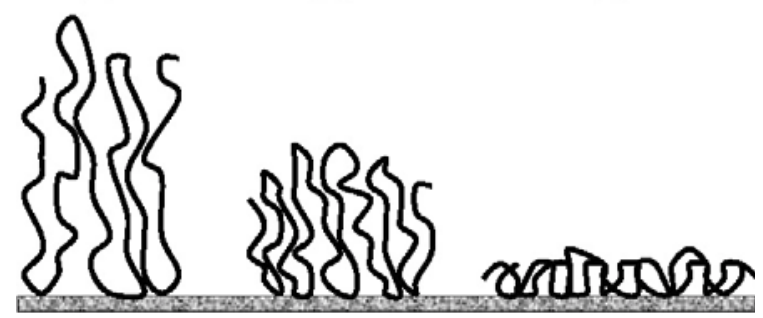

Fig. 3. Schematic of the absorption conformation of a single cationic polymer with counterions of different natures. a. PDADMAC; b. PDADMASO c. $\mathrm{PDADMANO}_{3}$.

adsorb uniformly on the mica surface with loops and tails (Fig. 3b). For the $\mathrm{PDADMANO}_{3}$ polymer, the larger size of the monovalent nitrate counterions leads to a higher cationic density in the polymer chain, a stronger intramolecular repulsive force and a more extended molecular chain, so that $\mathrm{PDADMANO}_{3}$ adopts a flat and layer-layer adsorption without loops and tails extended from the surface, and therefore develops a sponge-like structure (Fig. 3c).

\subsection{Solution properties of PDADMAX having different contents of $\mathrm{SO}_{4}^{2-}$ or $\mathrm{NO}_{3}^{-}$}

In the deionized water, reduced viscosity of PDADMAX having different contents of $\mathrm{SO}_{4}^{2-}$ or $\mathrm{NO}_{3}^{-}$was determined under the conditions that concentration was $1.65 \times 10^{-2} \mathrm{~mol} / \mathrm{L}$ and temperature was $25 \pm 0.1^{\circ} \mathrm{C}$. As shown in Fig. 4 , when the content of $\mathrm{SO}_{4}^{2-}$ was lower than $20 \%$, reduced viscosity of PDADMAX increased remarkably with increase of the $\mathrm{SO}_{4}^{2-}$ content. After $20 \%$, reduced viscosity of PDADMAX decreased rapidly with the constant increment of the $\mathrm{SO}_{4}^{2-}$ content. The cause of this phenomenon may be that $\mathrm{SO}_{4}^{2-}$ has bivalence counterions, which is strongly electronegative and has a strong connection action. When contents of $\mathrm{SO}_{4}^{2-}$ are lower than $20 \%$, the action of $\mathrm{SO}_{4}^{2-}$ is mainly inter-

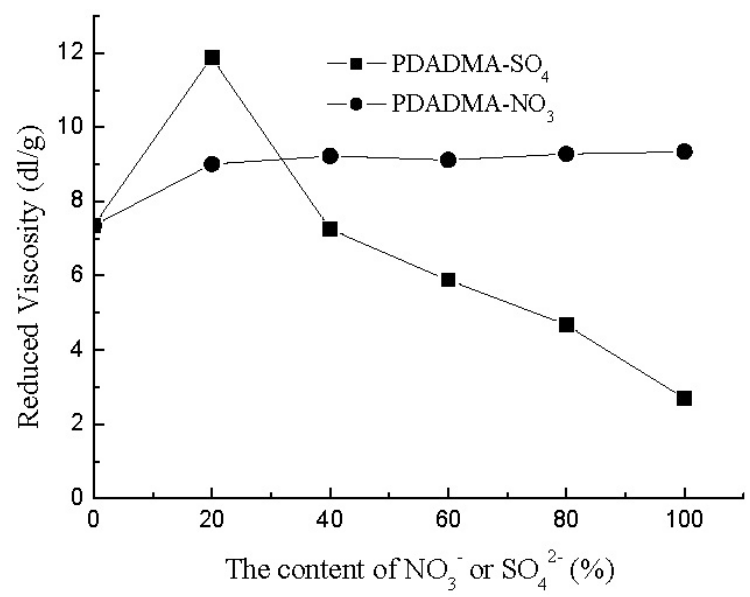

Fig. 4. Reduced viscosity (dl/g) of PDADMAX having different contents of $\mathrm{SO}_{4}^{2-}$ or $\mathrm{NO}_{3}^{-}$.

molecular, and therefore, reduced viscosity of PDADMAX increased rapidly. When the content of $\mathrm{SO}_{4}^{2-}$ is more than $20 \%$, the connection action of $\mathrm{SO}_{4}^{2-}$ gradually tends to intramolecular, so that the molecular chains of PDADMAX coil more. Finally, reduced viscosity of PDADMAX decreased quickly. On the other hand, as shown in Fig. 4, the influence of $\mathrm{NO}_{3}^{-}$upon reduced viscosity of PDADMAX is different. At the range of the low content of $\mathrm{NO}_{3}^{-}(<20 \%)$, the reduced viscosity of PDADMAX having $\mathrm{NO}_{3}^{-}$increased rapidly. After $20 \%$, the change of reduced viscosity became very little. The cause may be that the radius of $\mathrm{NO}_{3}^{-}$is bigger than that of $\mathrm{Cl}^{-}$ slightly $\left(\mathrm{NO}_{3}^{-}\right.$is a monovalent counterion so that it only has intramolecular action). The strength of coulombic interactions between $\mathrm{NO}_{3}^{-}$and the positive charged groups of the polyion was weaker than $\mathrm{Cl}^{-}$. So the positive electricity of PDADMAX having $\mathrm{NO}_{3}^{-}$is stronger so that the more extended molecular chain is obtained. Therefore, reduced viscosity of PDADMAX having $\mathrm{NO}_{3}^{-}$increases obviously. However, when the content of $\mathrm{NO}_{3}^{-}$continues to increase, this effect would be shielded, reduced viscosity of PDADMAX having $\mathrm{NO}_{3}^{-}$changes little. 
3.3. Flocculation experiments of the PDADMAX having different contents of $\mathrm{SO}_{4}^{2-}$ or $\mathrm{NO}_{3}^{-}$

\subsubsection{Flocculation experiments for $200 \mathrm{mg} / \mathrm{L}$} kaolin suspension

To clarify the influence of various contents of $\mathrm{SO}_{4}^{2-}$ or $\mathrm{NO}_{3}^{-}$counterions on the flocculation properties of PDADMAX, the flocculation experiment was carried out in $200 \mathrm{mg} / \mathrm{L}$ kaolin suspension solution at $\mathrm{pH}=7.53$ (the turbidity and temperature of the raw water is 198 NTU and $20^{\circ} \mathrm{C}$, respectively). The experiment results showed that all their optimal adding amounts are $0.1 \mathrm{mg} / \mathrm{L}$, using PDADMAX having various contents of $\mathrm{SO}_{4}^{2-}$ and $\mathrm{NO}_{3}^{-}$as flocculants. The measured values of residual turbidity (RT), zeta potential and flocculation index at the optimal flocculation point are shown in Fig. 5a-d, respectively. As shown in Fig. 5a, under the condition of low turbidity $(200 \mathrm{mg} / \mathrm{L}$ kaolin suspension solution), although the flocculation efficiency of all PDADMAX polymer is comparatively low, the turbidity removal increased evidently and both optimal contents of $\mathrm{SO}_{4}^{2-}$ or $\mathrm{NO}_{3}^{-}$are $20 \%$. The optimal RT $85.3 \mathrm{NTU}$ by PDADMAX having $\mathrm{NO}_{3}^{-}$is better than the optimal RT (91.2 NTU) by PDADMAX having $\mathrm{SO}_{4}^{2-}$. In Fig. 5b, it can be found that the zeta potential by PDADMAX is positive. The zeta potential by PDADMAX having $\mathrm{NO}_{3}^{-}$is higher than that by PDADMAX having $\mathrm{SO}_{4}^{2-}$. It is important that the maximum value of the zeta potential by both PDADMAX having $\mathrm{NO}_{3}^{-}$and PDADMAX having $\mathrm{SO}_{4}^{2-}$ is the counterion content of $20 \%$. After $20 \%$, the zeta potential by PDADMAX having $\mathrm{SO}_{4}^{2-}$ quickly decreased, while the zeta potential by PDADMAX having $\mathrm{NO}_{3}^{-}$only reduced slightly. As be seen in Fig. $5 \mathrm{c}$ and d, the FI curves by both PDADMAX having $\mathrm{SO}_{4}^{2-}$ and PDADMAX having $\mathrm{NO}_{3}^{-}$slowly increased step by step. All the values of FI are smaller than 1.0. Fig. $5 \mathrm{c}$ and $\mathrm{d}$ also showed that the maximum FI value by both PDADMAX having $\mathrm{SO}_{4}^{2-}$ and PDADMAX having $\mathrm{NO}_{3}^{-}$is at the point of $20 \%$.
However, the FI value by PDADMAX having $\mathrm{SO}_{4}^{2-}$ is bigger that that by PDADMAX having $\mathrm{NO}_{3}^{-}$.

\subsubsection{Flocculation experiments for $1000 \mathrm{mg} / \mathrm{L}$ kaolin suspension}

Under the same conditions, the flocculation experiments for $1000 \mathrm{mg} / \mathrm{L}$ kaolin suspended solution (the turbidity of the raw water is 980 NTU and $20^{\circ} \mathrm{C}$, respectively) by PDADMAX having various contents of $\mathrm{SO}_{4}^{2-}$ or $\mathrm{NO}_{3}^{-}$was undertaken, respectively. The optimal amount of PDADMAX to be added is $0.5 \mathrm{mg} / \mathrm{L}$. At the optimal flocculant dosage, the measured values of RT, zeta potential and FI are shown in Fig. 6a-d, respectively.

Using PDADMAX as flocculants under the condition of high turbidity $(1000 \mathrm{mg} / \mathrm{L}$ kaolin suspended solution), it can be found in Fig. 6a that turbidity removal obviously was improved and the RT became lower. Moreover, it was similar to under the condition of low turbidity that the optimal counterions content of $\mathrm{SO}_{4}^{2-}$ or $\mathrm{NO}_{3}^{-}$is $20 \%$. At the point, the optimal RT by PDADMAX having $\mathrm{NO}_{3}^{-}$was $21.5 \mathrm{NTU}$ and the turbidity removal was $98 \%$, while the optimal RT by PDADMAX having $\mathrm{SO}_{4}^{2-}$ was $43.5 \mathrm{NTU}$ and the turbidity removal was $96 \%$. In Fig.6b, the curves of the zeta potential showed that zeta potentials of the supernatant solution with both PDADMAX having $\mathrm{NO}_{3}^{-}$and PDADMAX having $\mathrm{SO}_{4}^{2-}$ were negative. Zeta potentials of the supernatant solution with PDADMAX having $\mathrm{NO}_{3}^{-}$were higher than that with PDADMAC. Its maximum value was the content of $20 \%$ and then reduced remarkably after this point. On the contrary, the zeta potential of the supernatant solution using PDADMAX having $\mathrm{SO}_{4}^{2-}$ was lower than that using PDADMAC. The minimal point also was the content of $20 \%$. In Fig. $6 \mathrm{c}$ and $\mathrm{d}$, it can be found that the FI curve was quickly increased to the maximum value under the condition of high turbidity. The FI values were 

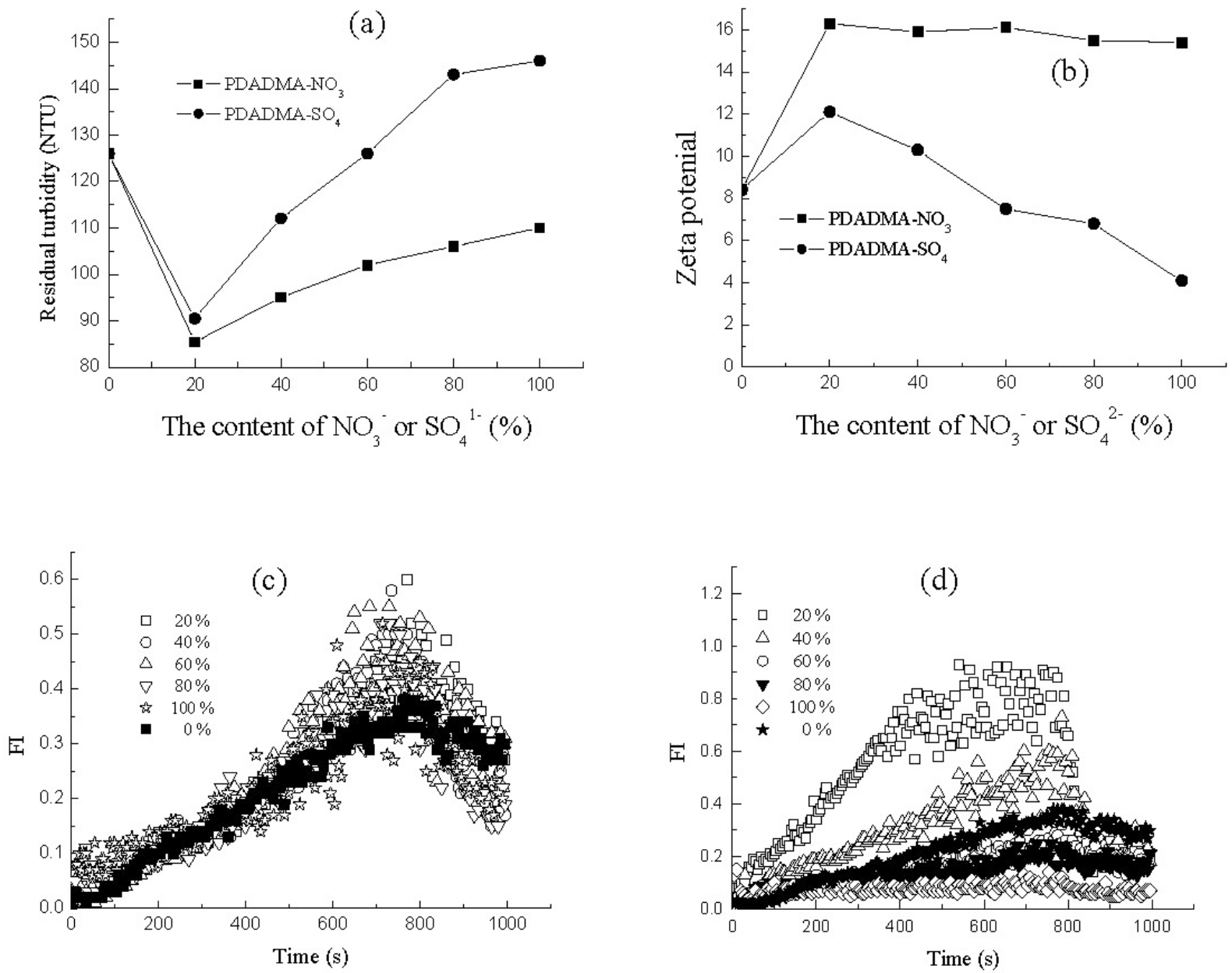

Fig. 5. Effects of different contents of $\mathrm{NO}_{3}^{-}$or $\mathrm{SO}_{4}^{2-}$ on the flocculation for $200 \mathrm{mg} / \mathrm{L}$ kaolin suspensions at $\mathrm{pH}=7.53$ by using PDADMAX as flocculants: (a) residual turbidities; (b) zeta potential; (c) flocculation index of PDADMAX having $\mathrm{NO}_{3}^{-}$; (d) flocculation index of PDADMAX having $\mathrm{SO}_{4}^{2-}$.

obviously improved and the content of both $\mathrm{NO}_{3}^{-}$ and $\mathrm{SO}_{4}^{2-}$ counterions at the maximum values was $20 \%$. The maximum values by PDADMAX having $\mathrm{NO}_{3}^{-}$was $3.5-4.0$, while the maximum values by PDADMAX having $\mathrm{SO}_{4}^{2-}$ were higher (5.0-6.0).

The results of the flocculation test indicated that the flocculation efficiency can be improved by modifying the PDADMAX polymer with counterions. Using $\mathrm{NO}_{3}^{-}$or $\mathrm{SO}_{4}^{2-}$ as counterions, the optimal counterions content is $20 \%$. However, the flocculation mechanism by PDADMAX having $\mathrm{NO}_{3}^{-}$is different from that of PDADMAX having $\mathrm{SO}_{4}^{2-}$. These phenomena can be explained that PDADMAX having $\mathrm{NO}_{3}^{-}$have the higher charged density and the more extended molecular train (see Fig. 4). The maximum value is the content of $20 \%$. It adopts a flat and layer-layer adsorption without "loops and tails" extended from the surface (see Figs. 2 and 3). So it has a stronger "charge neutralization action" than PDADMAC in the flocculation of the kaolin 

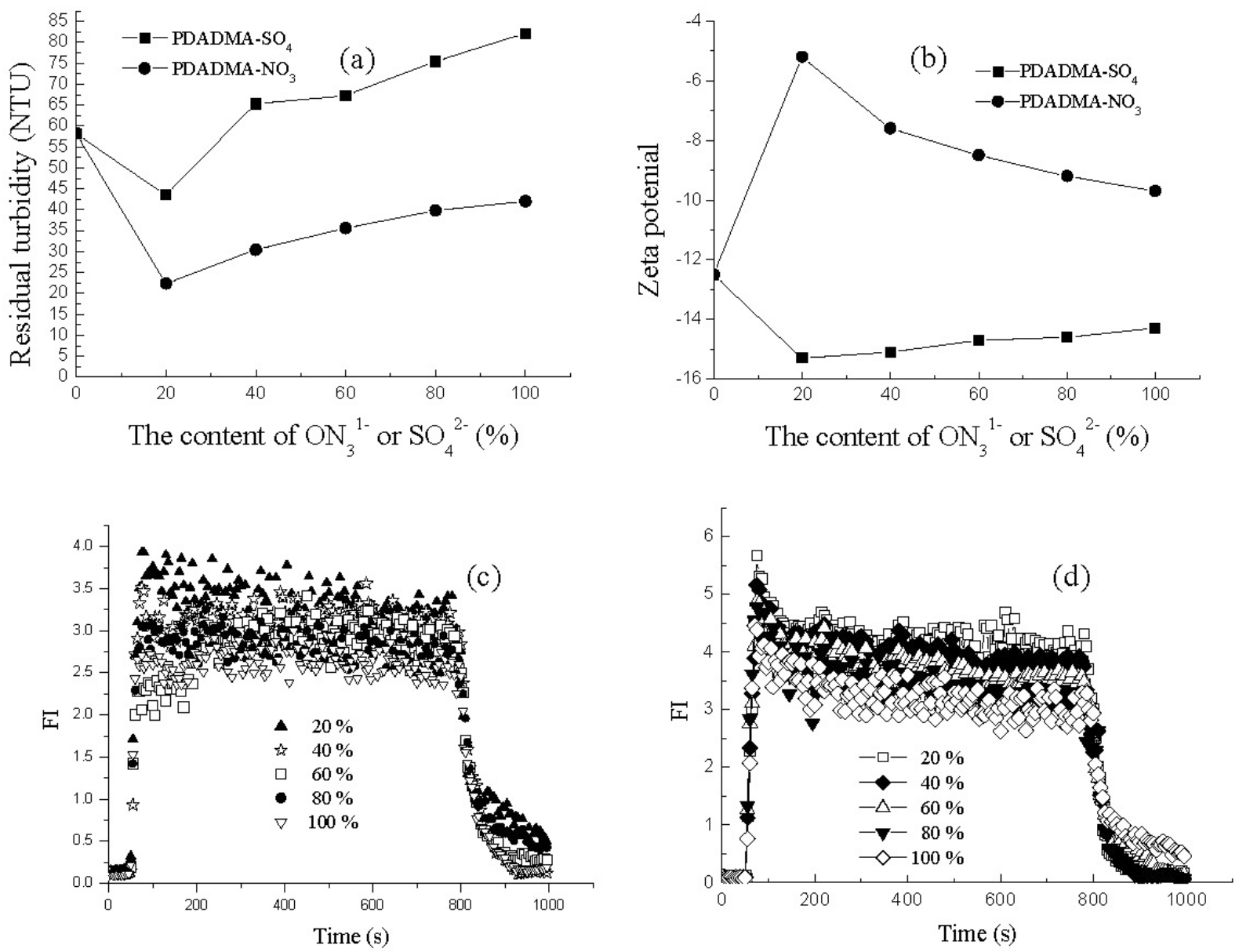

Fig. 6. Effects of different contents of $\mathrm{NO}_{3}^{-}$or $\mathrm{SO}_{4}^{2-}$ on the flocculation for $1000 \mathrm{mg} / \mathrm{L}$ kaolin suspensions at $\mathrm{pH}=7.53$ by using PDADMAX as flocculants: (a) residual turbidities; (b) zeta potential; (c) flocculation index of PDADMAX having $\mathrm{NO}_{3}^{-}$; (d) flocculation index of PDADMAX having $\mathrm{SO}_{4}^{2-}$.

suspension (see Figs. 5b,c and 6b,c). However, PDADMAX having $\mathrm{SO}_{4}^{2-}$ may have more coil molecular chains and the maximum value of the FI value also is the content of $20 \%$. The reason is that $\mathrm{SO}_{4}^{2-}$ was a bivalue counterion. It has the intermolecular attractive force when the content of $\mathrm{SO}_{4}^{2-}$ was $20 \%$ (see Fig. 4). It adsorbs with "loops and tails" at the oppositely charged particle surface (see Figs. 2 and 3). It has stronger "adsorption bridging action" (see Figs. 5b,d and $6 b, d)$.
The flocculation results in Figs. 5 and 6 also implied that under the condition of low turbidity (200 mg/L kaolin suspension solution), the flocculation mechanism of PDADMAX polymer seems to be mainly "adsorption charge neutralization action". It can be proved by the AFM image under high concentration (a flat and layerlayer adsorption; Fig. 2a,c,e). According to the flocculation theory, such a kind of adsorption structure and conformation is liable to lead to an over-coverage adsorption and a poor flocculation 
performance. Though the modified PDADMAX polymer increased the flocculation efficiency, RT still is high (80-90 NTU) and the FI value is smaller than 1.0. When the concentration of the kaolin suspended solution was improved to $1000 \mathrm{mg} / \mathrm{L}$, with increase of the turbidity, the flocculation efficiency of PDADMAX polymer was obvious improved. This phenomenon suggested that "adsorption bridging action" seems to be that main flocculation mechanism under the condition of high turbidity so that the flocculation efficiency of the cationic polymer flocculant was obviously improved. It also can be proved by the AFM image under low concentration ("pools and tails" adsorption structure, in Fig. 2b,d,f). Under the high turbidity conditions, PDADMAX having $\mathrm{NO}_{3}^{-}$have not only strong "adsorption bridging action", but also have a stronger "adsorption charge neutralization action", and therefore, has a higher turbidity removal (98\%) and lower RT (21.5 NTU). For PDADMAX having $\mathrm{SO}_{4}^{2-}$, it can be found that it reduces "adsorption charge neutralization action" and increases "adsorption bridging action", so that it has larger floc size and wider optimum dosage.

\section{Conclusions}

The experiment results show that a counterion has a significantly different effect on PDADMAX flocculation where "adsorption charge neutralization action" and "adsorption bridging action" play different parts. Compared with PDADMAC, PDADMAX having $\mathrm{NO}_{3}^{-}$has stronger flocculation efficiency, whereas PDADMA having $\mathrm{SO}_{4}^{2-}$ has the wider optimum dosage and larger floc size. Although the flocculation efficiency of PDADMAX having different content of $\mathrm{SO}_{4}^{2-}$ or $\mathrm{NO}_{3}^{-}$is different, the optimum content of $\mathrm{SO}_{4}^{2-}$ or $\mathrm{NO}_{3}^{-}$is $20 \%$. In particular, for high turbidity kaolin suspension solution, the flocculation of PDADMAX having $\mathrm{SO}_{4}^{2-}$ or $\mathrm{NO}_{3}^{-}$is obviously increased.
It is interesting that in contrast with the more extended polymer chain of PDADMANO 3 , both the floc size and the settling rate of $\mathrm{PDADMNO}_{3}$ are smaller than those of $\mathrm{PDADMASO}_{4}$. These results seem to be paradoxical with the higher reduced viscosity in aqueous solution of $\mathrm{PDADMNO}_{3}$. The problem can be explained that the effect of $\mathrm{SO}_{4}^{2-}$ and $\mathrm{NO}_{3}^{-}$on the flocculation mechanisms of PDADMAX is different, which can be proved from the AFM images of polymer conformation and absorbed layer structures. It can be concluded that $\mathrm{PDADMANO}_{3}$ adopts a flat and layer-layer adsorption without "loops and tails" extended from the surface so that the kaolin suspension was mainly flocculated via the adsorption charge neutralization action mechanism, whereas $\mathrm{PDADMASO}_{4}$ adsorbs with "loops and tails" at the particle surface so that the adsorption bridging action mechanism can be involved.

\section{Acknowledgements}

We are grateful for financial support from the National 10th Five-year Science and Technology Project of China (No. 2002BA806b04-01B) and the National High-Tech R\&P Program (863) of China (2002AA601310).

\section{References}

[1] M. Sotiropoulou, C. Cincu and G. Bokias, Polymer 45 (2004) 1563.

[2] G. Marcelo, M.P. Tarazona and E. Saiz, Polymer, 45 (2004) 1321.

[3] M. Möller and E. Nordmeier, Eur. Poly. J., 38 (2002) 445.

[4] B.H. Tian and Z.K. Luan, Radiation Physics Chem., 73 (2005) 328.

[5] A. Matsumoto, Prog. Polym. Sci., 26 (2001) 189.

[6] A.B. Brain, Prog. Polym. Sci., 20 (1995) 987.

[7] H.Z. Zhao, Z.K. Luan and B.Y. Gao, J. App. Polym. Sci., 84 (2002) 335.

[8] Q. Chang, Y. Chen and B.H. Tian, J. Environ. Sci., $20(2000) 168$. 
[9] G. Petzold and K. Lunkwitz, Colloids Surf. A: Physicochem. Eng. Aspects, 98 (1995) 225.

[10] S.Z. Zhang, K.C. Gong and J.W. Lu, Mat. Lett., 58 (2002) 1292.

[11] M. Zhu, M. Schnelder and G. Papastavrou, Langmuir, 17 (2001) 6471.

[12] E. Kokufuta, S. Kokulo and S. Iwai, Nippon Kagaku Kaishi, 49 (1976) 1335.

[13] E. Kokufuta, S. Kokulo and S. Iwai, Shikizai Kyokaishi, 49 (1976) 589.

[14] L.M. Bowman and C.Y. Cha, J. Poly. Sci., Poly. Lett. ed., 17 (1779) 167-176.

[15] D. Hinderberger, G. Jeschke and H.W. Spiess, Macromolecules, 35 (2002) 9698.

[16] M. Kawaguchi and A. Takahashi, Adv. Colloid Interf. Sci., 37 (1992) 219.

[17] M. Kawaguchi, W. Saito and T. Kato, Macromolecules, 27 (1994) 5882.

[18] J.J. Harris and M.L. Bruening, Langmuir, 16 (2000) 2006.

[19] M. Losche, J. Schmitt and G. Decher, Macromolecules, 31 (1998) 8893.
[20] V.V. Balastre, A.L. Tolstikhina and N.D. Stepina, Cryst. Rep., 43 (1998) 124.

[21] M. Balastre, J. Persello and A. Foissy, J. Coll. Interf. Sci., 219 (1999) 155.

[22] J.A. He, R. Valluzzi and K. Yang, Chem. Mat., 11 (1999) 3268.

[23] L.C.D.S. Maria, A.P.D. Aguiar and M.R.M.P. Aguiar, Mat. Lett., 58 (2004) 563.

[24] A. Minakata, H. Takahashi, T. Nashio and J. Nagaya, Coll. Surf. A: Physicochem. Eng. Aspects, 209 (2002) 213.

[25] C. Wandrey,W. Jaeger and G. Reinisch, Acta Polym., 33 (1982) 156.

[26] K.J. Nishida, K. Kaji and T. Kanaya, Polymer, 43 (2002) 1295.

[27] D.S. Wang, H.X. Tang and J. Gregory, Environ. Sci. Technol., 36 (2002) 1815.

[28] K. Benmansour, K. Medjahed, L. Tennouga and A. Mansri, Eur. Polym. J., 39 (2003) 1443.

[29] B.L. Rivas, E.D. Pereira and A. Horta, Eur. Polym. J., 40 (2004) 203. 